\title{
Two-phase Multicast in Wormhole-Switched Bidirectional Multistage Banyan Networks ${ }^{\dagger}$
}

\author{
W. Kwon \\ CSD KAIST \\ 373-1 Kusong-Dong, \\ Yusong-Gu,Taejon \\ 305-701, Korea \\ wnkwon@camars. \\ kaist.ac.kr
}

\author{
B. Kwon \\ CEED \\ Andong National Univ. \\ 388 Songchun-Dong, \\ Andong, Kyung-Book \\ 760-380, Korea \\ bskwon@camars. \\ kaist.ac.kr
}

\author{
J. Park \\ CSD Purdue Univ. \\ West Lafayette, \\ IN, 47907, USA \\ hyeoung@cs. \\ purdue.edu
}

\author{
H. Yoon \\ CSD KAIST \\ 373-1 Kusong-Dong, \\ Yusong-Gu,Taejon \\ 305-701,Korea \\ hyoon@camars. \\ kaist.ac.kr
}

\begin{abstract}
A multistage interconnection network is a suitable class of interconnection architecture for constructing large-scale multicomputers. Broadcast and multicast communication are fundamental in supporting collective communication operations such as reduction and barrier synchronization. In this paper, we propose a new multicast technique in wormhole-switched bidirectional multistage banyan networks for constructing large-scale multicomputers. To efficiently support broadcast and multicast with simple additional hardware without deadlock, we propose a two-phase multicast algorithm which takes only two transmissions to perform a broadcast and a multicast to an arbitrary number of desired destinations. We encode a header as a cube and adopt the most upper input link first scheme with periodic priority rotation as arbitration mechanism on contented output links. We coalesce the desired destination addresses into multiple number of cubes. And then, we evaluate the performance of the proposed algorithm by simulation. The proposed two-phase multicast algorithm makes a significant improvement in terms of latency. It is noticeable that the two-phase algorithm keeps broadcast latency as efficient as the multicast latency of fanout $2^{m}$ where $m$ is the minimum integer satisfying $2^{m} \geq \sqrt{N}$ ( $N$ is a network size $)$.
\end{abstract}

\section{Introduction}

Multistage interconnection networks(MINs) are a popular class of interconnection architecture for constructing

\footnotetext{
${ }^{\dagger}$ This work is supported by network technology project of Ministry of Information and Commmunication Republic of Korea in 1998
}

scalable multicomputers, such as TMC CM-5[5], IBM SP1[1], and Meiko CS-2[7]. Efficient data communication among processing nodes is critical to the performance of message-based multicomputers [10]. In most existing MINbased multicomputers, wormhole-switching[6] which is designed for low latency and high performance has been adopted. However, the wormhole-switching is susceptible to deadlock due to the resource competition among multiple worms. Once deadlock occurs, the performance degrades quickly and remarkably. Hence, deadlock avoidance is an important issue for improving performance in these switching networks[6].

Multicast techniques that deliver a packet from a source to multiple destinations in wormhole-switched bidirectionalMINs have been studied. U-min algorithm [10] and C-min algorithm [3] are software techniques and don't cause deadlock. But, it takes at least $\lceil\log d\rceil(d$ is the number of destinations) number of setups and transmissions for these algorithms to perform a multicast. [2] proposes a multicast technique on central-buffer-based and input-queue-based switch architectures. This technique adopts buffered wormholeswitching which stores blocked flits in buffers in switching elements, and asynchronous replication which allows flits of a multicast packet to be transmitted to the successfully reserved subset of requested output links. Moreover, this technique requires only one setup and transmission for a multicast and does not cause deadlock. Therefore, this technique can support multicast efficiently. However, the hardware complexity of the switch architectures is very high because the architectures must contain input queues or a central buffer of large space to avoid deadlock and to get desired performance gain. Therefore, this technique has difficulties in constructing large-scale multicomputer systems [9].

In this paper we propose a new multicast algorithm in 
pure wormhole-switched bidirectional multistage banyan networks without central buffers nor queues. The proposed multicast algorithm is a software technique, which is supported by one-flit output buffers and simple replication hardware. The multicast algorithm consists of two phases : phase I and II. In phase I, a multicast packet is copied from a source through a network to computed destinations. In phase II, the destinations generated in phase I send the received multicast packets to the desired destinations of the multicast. The multicast algorithm employs cube-encoding as header encoding and adopts the most upper input link first scheme with periodic priority rotation as arbitration mechanism among multiple multicast packets to avoid deadlock. The proposed algorithm requires only 2 transmissions to achieve a multicast. Hence, we call this multicast technique two-phase (multicast) algorithm. The architecture is so simple to be suitable for constructing large-scale multicomputer systems, and two-phase algorithm can support efficient multicast and broadcast in large-scale multicomputer systems.

The remainder of this paper is organized as follows. The next section describes the system architecture and section 3 describes our two-phase multicast algorithm. We evaluate the performance of the proposed algorithm in section 4 and conclude this paper in section 5 .

\section{System Architecture}

We assume $N \times N$ bidirectional banyan networks constructed by $2 k \times 2 k$ switching elements. Figure 1 shows a

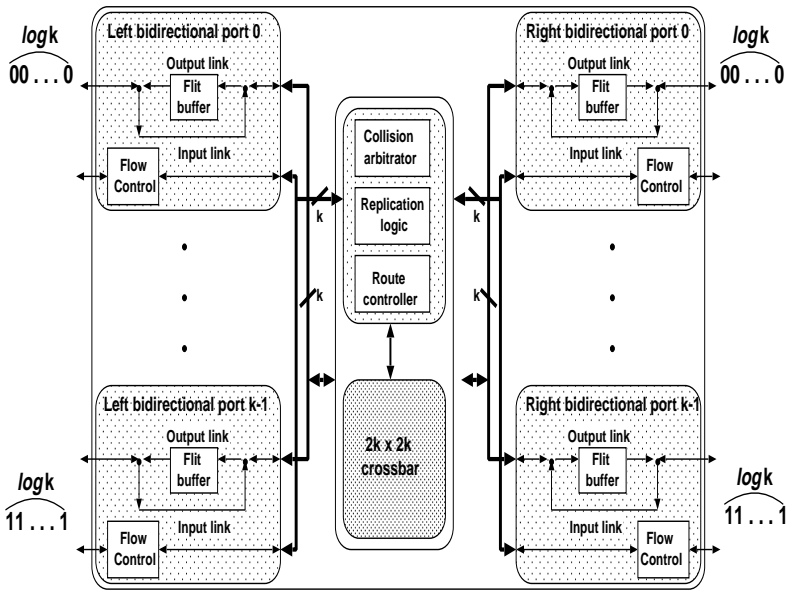

Figure 1. A $2 k \times 2 k$ bidirectional switching elment

$2 k \times 2 k$ switching element which consists of a $2 k \times 2 k$ crossbar and $2 k$ bidirectional ports. Unidirectional arrows and bidirectional arrows denote unidirectional links and bidirectional links, respectively. Each port can be implemented by two unidirectional ports with an input link and an output link. Notice that a switching element has an one-flit output buffer per bidirectional port. The addresses of bidirectional ports are represented as binary numbers between $\overbrace{0 \ldots 0}^{\log k} \overbrace{1 \ldots 1}^{\log k}$

$\overbrace{0 \cdots 0}$ and $\overbrace{1 \cdots 1}^{0}$. A bidirectional port can send and receive flits simultaneously.

The network employs a pure wormhole-switching technique without input queues nor central buffers. And it adopts synchronous replication because it is difficult for asynchronous replication to get up desired performance without large buffer. The replicated flits of a multicast are transmitted only when none of replicated flits of the multicast packet is blocked. Otherwise, all of them is blocked in place. Synchronous replication is controlled by flow control logic. Flow control logics communicates feedback control information one another to synchronize transmission of a multicast packet.

In this network, after the flits of a multicast packet are transmitted adaptively up to the least common ances$\operatorname{tor}(\mathrm{LCA})$ stage [4] of the source and its destinations, they synchronously replicate downwards on the way back from the LCA stage to reach the destinations. Assume that all processing elements are in the left side of the network connecting them, like figure 3. A switching element replicates and routes incoming flits to output links by using the crossbar and the hardware replication logic therein. When a header flit arrives at a input link, the switching element routes it to the ouput buffer(s) of detined output port(s). If a header flit is forwarding up to its LCA stage, the switch element routes it to the output buffer of an available most upper right bidirectional port. If a header flit is at its LCA stage or is backwarding down to its destination processing node, the switching element routes or replicates and routes it to the output buffer(s) of the left bidirectinal port(s) determined by destination tag routing.

\section{Two-Phase Multicast Algorithm}

\subsection{Multicast Packet Format}

The formats of multicast packets are shown in Figure 2. Each multicast packet of phase I and II consists of a header flit and following data flits. The header flits of phase I and II contain the destination tags for their own destinations. The destinations of phase I and II are called intermediate destinations and desired destinations, respectively. Notice that in the multicast packet of phase I the information of desired destinations is placed in front of the series of data to transmit. At the end of the information of desired destinations, EOD(End-Of-Destination) flit is put because the number of desired destinations is variable. In the ends of both multicast 


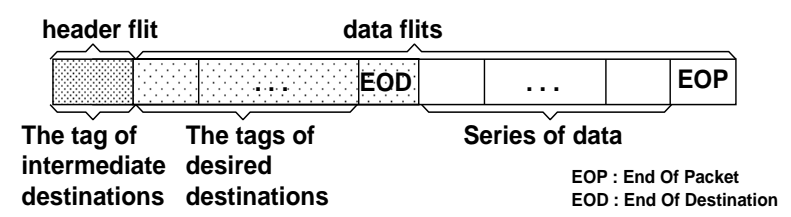

(a) The multicast packet format in phase I

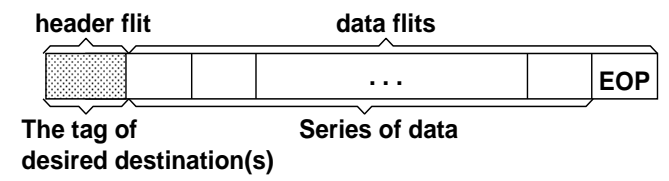

(b) The multicast packet format in phase II

\section{Figure 2. The packet formats of phase I and II in two-phase multicast}

packets, EOP(End-Of-Packet) flits are appended to mark the end of multicast packets.

\subsection{Header Encoding}

We encode headers as $k$-ary cubes defined in below definition. A $k$-ary cube is represented as the corresponding binary cubes. The binary representations of $k$-ary cubes are

Definition $k$-ary cube :

$k$-ary digit is $d, y$, or $X$ where

$d$ is a number satisfying $0 \leq d \leq k-1$,

$y$ is a binary cube $b_{\log k-1} \cdots b_{m} \overbrace{x \cdot x}^{m}$

where for $m$ and $j$ satisfying $0 \leq m \leq j \leq \log k-1$, $b_{j} \in\{0,1\}$ and $x$ is a don't care, and

$X$ is $\overbrace{x \cdots x}^{\log k}$.

$k$-ary cube of size $2^{m} k^{c}$ is defined as

$$
\begin{aligned}
& d_{n-1} \cdots d_{c+1}(y) \overbrace{X \cdots X}^{c}(0 \leq c \leq n), \\
& \quad \text { where } d_{i}(c+1 \leq i \leq n-1) \text { is a number } \\
& \quad \text { satisfying } 0 \leq d_{i} \leq k-1 .
\end{aligned}
$$

used for replicating and routing flits in switching elements. For example, when $n=3$, all 4-ary digits from 220 to 233 constitue a 4 -ary cube $2(1 x) X$. The cube size is $2^{3}$ and the binary representation is $101 x x x$.

\subsection{Multicast Algorithm}

We propose a multicast algorithm, called two-phase multicast algorithm, which consists of phase I and phase II. Before both phases, every multicast packet is re-constructed into a header flit and following data flits. In order to perform a multicast to multiple desired destinations, in phase I a multicast packet is copied from a source through a network to computed intermediate destinations, and then, in phase II all or some of intermediate destinations transmit the received multicast packets to the corresponding desired destinations.

Algorithm Phase I : a multicast at a source $s$

Input :

$$
\begin{aligned}
& D=\left\{D_{0}, \ldots, D_{d-1}\right\}: \text { a sorted list of desired destina- } \\
& \text { tion addresses } \\
& p: \text { data to send }
\end{aligned}
$$

Procedure :

$$
C\left(=\left\{C_{0}, \ldots, C_{c-1}\right\}\right) \leftarrow \operatorname{Encode}(D) ;
$$

Calculate the minimum integer $c$ such that $2^{c} \geq|C|$;

Select a $k$-ary cube $H$ of size $2^{c}$;

Construct a multicast packet $P(=H|C| E O D|p| E O P)$; Send $P$ to the intermediate destinations $H$;

\section{End}

The setup algorithms of phase I and II are shown in algorithm Phase I and Phase II, respectively. Before a multicast, in phase I, a source computes the set of the tags of its desired destinations $(C)$ with Encode algorithm to be explained in the next section, and then encodes the header of phase I as a $k$-ary cube $(H)$ with the smallest size that is power of two and greater than the number of elements in $C$. After this, the source constructs its multicast packet by writing $H$ on a header flit, and $C$ and data $p$ on data flits, like Figure 2.(a). The setup of phase I terminates by starting to push the multicast packet $P$ into the network.

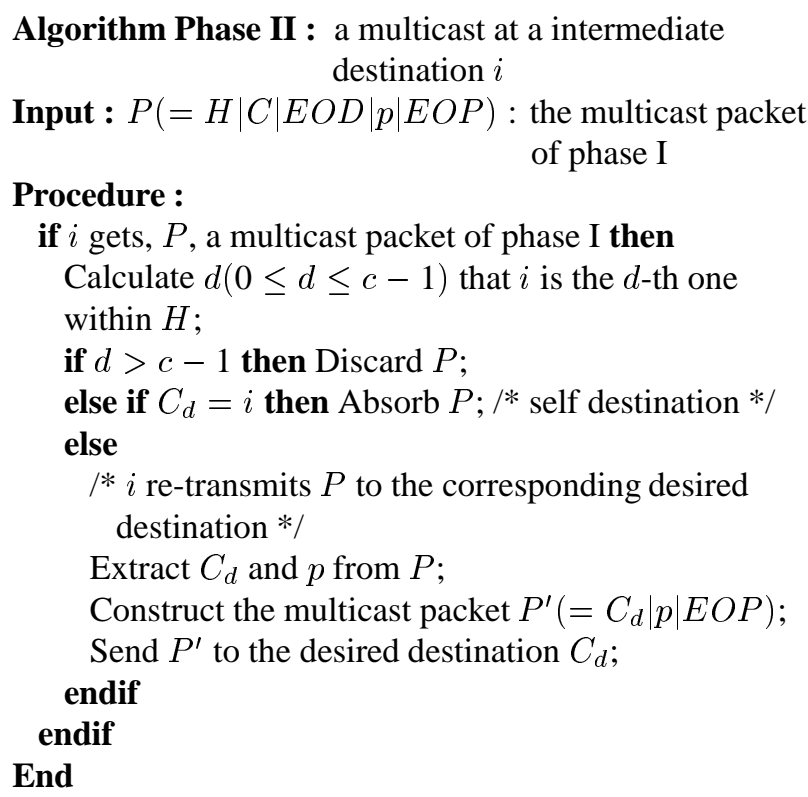

Phase II begins after an intermediate destination receives the packet $P$ of phase I. In the setup of phase II, the $d$-th intermediate destination extracts the $d$-th desired destination tag $C_{d}$ and data $p$ from the received packet $P$. It re-constructs its multicast packet by writing $C_{d}$ on a header flit and $p$ on 
data flits, like Figure 2-(b). After that, it terminates and the source starts to transmit $P^{\prime}$.

\subsection{Encode Algorithm}

Encode algorithm may be simply designed to do no operation or just sort the desired destinations. As the number of desired destinations becomes more, the fanout of phase I becomes larger, the blocking probability gets higher, and consequently latency becomes longer. At this, we propose Coalesce algorithm as Encode algorithm to decrease the fanout of phase I. Coalesce algorithm coalesces the desired destination addresses into multiple $k$-ary cubes, as shown in below algorithm. In the algorithm, a maximal $k$-ary cube means the cube has the greatest size among $k$ ary cubes that the subeset of $D$ can construct. This al-

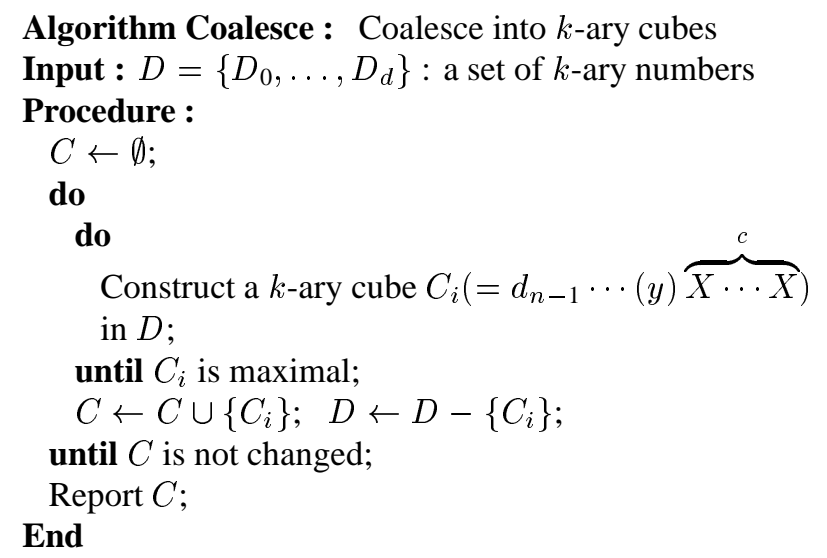

gorithm keeps multicast fanout values under the half of network size. For example, Encode coalesces a set of 4-ary numbers, $\{00,02,03,12,22,23,30,31,32,33\}$, into $\{00,0(1 x), 12,2(1 x), 3 X\}$ with Coalesce algorithm.

\subsection{Broadcast}

The proposed two-phase algorithm with Coalesce algorithm has trouble to perform a broadcast, because the fanout of phase II is same with the size of the network wherein the broadcast takes place. To keep the fanout of broadcast in both phase I and II as small as possible, Encode partitions $N$ desired destinations into $k$-ary cubes with below Partition algorithm. Partition algorithm partitions the destination addresses so that the sum of cube sizes of phase I and II is minimum. For example, if $N=256$, Encode partitions 256 desired destination addresses into $16 k$-ary cubes of size 16 with Paritition algorithm.

\subsection{Deadlock Avoidance}

Two-phase multicast algorithm suffers from potential deadlock due to multiple multicast packets in a network. In
Algorithm Partition : Partition into $2^{m} k$-ary cubes of size $2^{l}$

Input : $N\left(=2^{n}\right)$

Procedure :

Compute the maximum integer $l$ such that $l \leq n / 2$;

Compute the minimum integer $m$ such that $m \geq n / 2$;

Construct $2^{m} k$-ary cubes of size $2^{l}$ from $\mathrm{N}$;

Report the constructed cubes;

End

multistage banyan networks using distributed control, one replicated flit of a multicast packet does not know whether other flits of the multicast packet are blocked or not. As a result, contention on output links can cause deadlock. Therefore, to avoid deadlock by eliminating circular waiting, we present the most upper input link first scheme as arbitration mechanism on contended output links. This scheme gives priority to the flit from the most upper input link, to reserve the desired output links, when contention occurs on same output links. The most upper input link first scheme guarantees a deadlock-free transmission through multistage banyan networks. In order to prevent starvation, priority rotates periodically. This arbitration mechanism is called the most upper input link first scheme with periodic priority rotation.

\subsection{An Example of Two-Phase Multicast Algo- rithm}

In a $16 \times 16$ bidirectional cube network where $8 \times 8$ switching elements are connected with butterfly permutation, a source 10 begins to transmit a multicast packet to its desired destinations, 00,02, 03, 12, 22, 23, 30,31, 32, and 33 with two-phase multicast algorithm, as shown in figure 3 . In phase I, the source coalesces the desired destinations into $00,0(1 x), 12,2(1 x), 3 X$ and computes a 4-ary cube of size $8,(1 x) X$, at random. And then, the source constructs the corresponding multicast packet and transmits it to $(1 x) X$. All intermediate destinations receive the multicast packet of phase I and phase II begins. In phase II, after re-constructing the multicast packet of phase II, intermediate destinations $20,21,22,23$, and 31 transmit their multicast packets to desired destinations $00,0(1 x), 12,2(1 x)$, and $3 X$, respectively. Other intermediate destinations discard their received packets.

\section{Performance Evaluation}

To assess the performance of two-phase multicast algorithm, we implemented and compared our algorithm and the binomial tree-based u-min[10] with several set of parameters. We did not compare two-phase algorithm with 
Source : 10

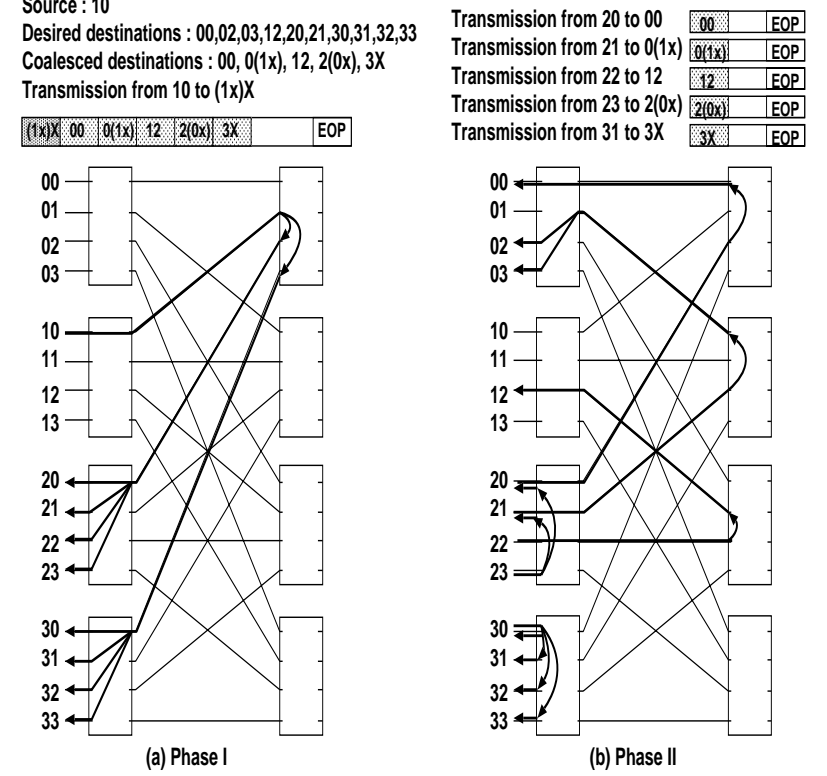

\section{Figure 3. An example of two-phase multicast algorithm with Coalesce algorithm}

[2] because of their architectural differences such as pure wormhole-switching vs. buffered wormhole-switching, and an one-flit output buffer per bidirectional port vs. one or more buffers of large space per switching element.

\subsection{Simulation Issues and Parameters}

Multicast traffic evaluation raises several additional issues beyond unicast traffic. First, multicast latency can be defined as the latency of the last received packet of a multicast, or as the average of the latency of each received packet of a multicast. The former definition is more important term in assessing packet-passing collective communication performance[8] and in assessing the performance of multicast used for cache line invalidations in shared-memory systems. Therefore, we chose the former one as latency.

Second, the load of a network injected by multicast packets can be defined as injected load or effective load. Because the effective load of our multicast algorithm cannot be estimated intuitively, we chose injected load as network load and defined the load as the viewpoint of the interarrival time of packets.

Finally, while a unicast has negligible startup overhead, the startup overhead of a multicast cannot be ignored due to multicast packet construction latency. We assume that startup latency is $2 \log N$ like in [10].

We assume 2-byte flit and count latency by the cycle in which a flit goes through a switching element. We simulated random traffic of 32-byte data packets. For simulating multicast of fanout $F$ in an $N$-node system, we randomly chose $F$ uniformly distributed destinations for each multicast packet. Packet arrival time, $I$, for each node were exponentially distributed. Measured latency includes queuing time at source nodes. To get stable environments for statistics collection, we ran the simulations with 50,000 wormup cycles before collecting statistics data. We simulated for bidirectional cube networks whose $2 K \times 2 K$ switching elements are connected by butterfly permutations, We collected statistics when $N=256, K=4$ and 16, and $F=16,64,128,192,240$, and 256 , and when $N=64$, $K=4$, and $F=16,32,48$, and 64 .

\subsection{Simulation Results}

The simulation results of two-phase multicast algorithm are shown in figure 4. Figure 4.(a) and (b) compare twophase algorithm with u-min algorithm. Figure 4.(a) shows that the latency of two-phase algorithm is much less than that of u-min and increases less dramatically. And figure 4.(b) compares the impacts of fanout on the latency of two-phase and u-min algorithm. As $F / N$ grows greater, the latency ratio of two algorithms increases. As a result, two-phase algorithm improves the performance at least about 200 percent than u-min algorithm from the viewpoint of latency.

Figure 4.(c) shows the impact of fanout on latency of two-phase algorithm with and without Coalesce. The doted lines show that without Coalesce, the latency of multicast to more than $N / 2$ desired destinations increases seriously and abruptly. This is because the blocking probability becomes greater due to the large fanout of phase I. However, as the solid lines show, with Coalesce the latency of multicast is bounded even when fanout becomes greater than $N / 2$. This is because Coalesce keeps the fanout values of multicasts same with or less than $N / 2$. In other words, if $F / N$ is over some value, the coalesceing effect increases and the latency decreases.

Figure 4.(d) shows multicast packet overhead defined as the total number of header flits, flits of desired destination tags, EOD flits, and EOP flits which have traversed through a network during a multicast. The multicast packet overheads of two-phase algorithm is less than that of u-min. And, as fanout value becomes greater, the overheads of $\mathrm{u}$-min grows greater, but that of two-phase algorithm is bounded. This is because fanout decreases due to coalescing effects when the number of desired destinations grows above some value.

Figure 4.(e) shows that the size of switching elements, $8 \times 8$ and $32 \times 32$, impacts little on the latency of varing fanout. Figure 4.(f) shows the impact of network size on latency. The graphs of different network size are similar in shape but the latency in smaller networks is less than that in larger ones due to the smaller number of stages of networks.

It is noticeable that the broadcast latency of two-phase 


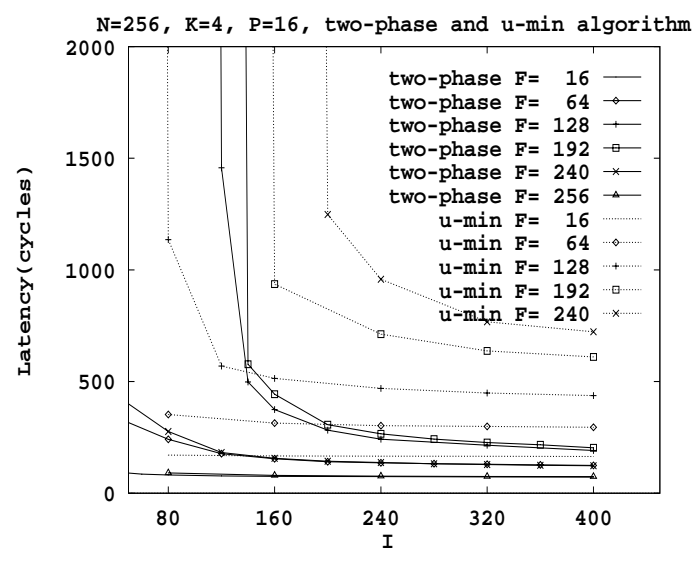

(a) Impact of interarrival time : latency vs. interarrival time for two-phase and u-min multicast algorithm

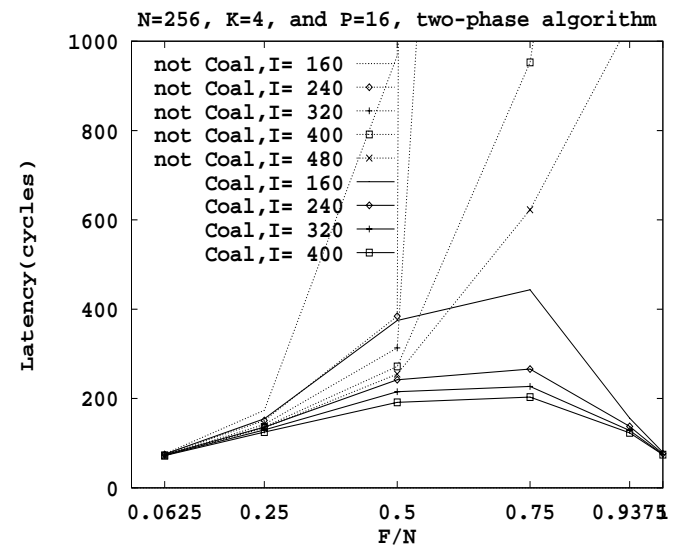

(c) Impacts of Coalesce : latency vs. (fanout)/(network size) for two-phase multicast algorithm

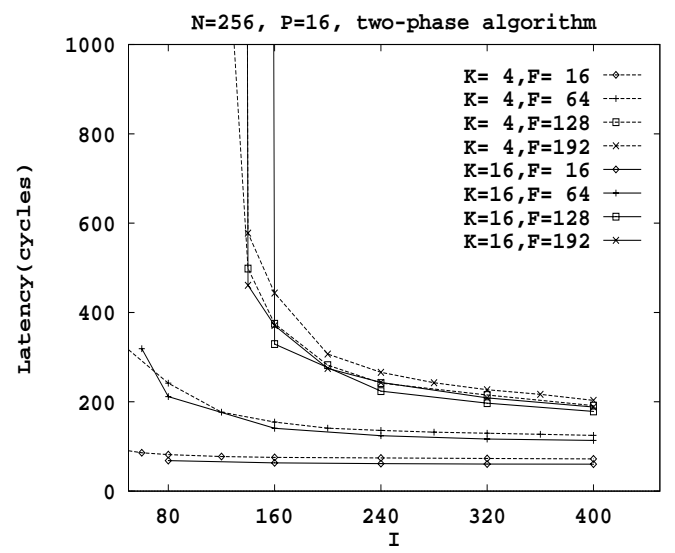

(e) Impacts of a switching element size : latency vs. fanout for two-phase multicast algorithm

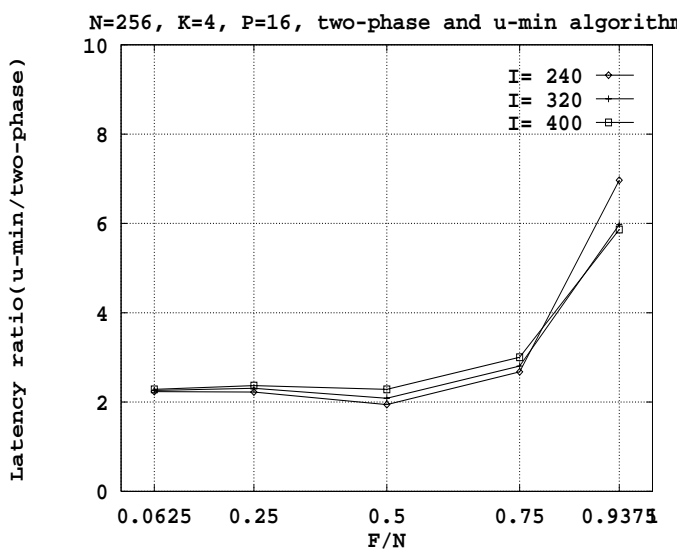

(b) Impact of fanout : the latency ratio of (two-phase)/(umin) vs. (fanout)/(network size) for two-phase and umin multicast algorithm

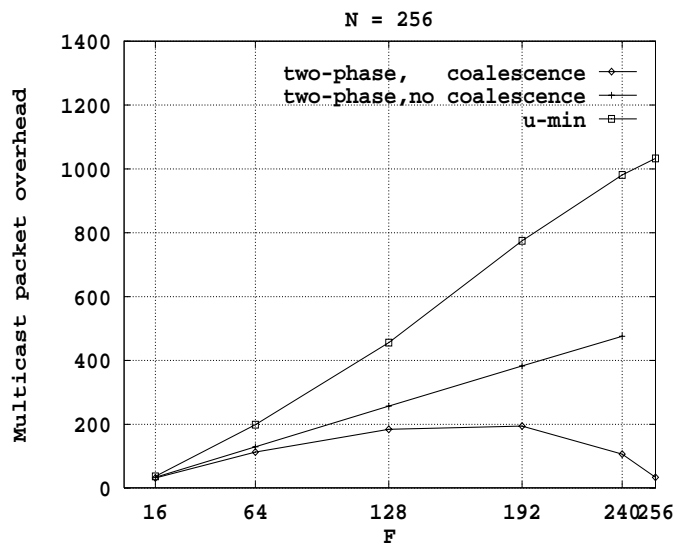

(d) Multicast packet overhead vs. fanout for two-phase and u-min multicast algorithm

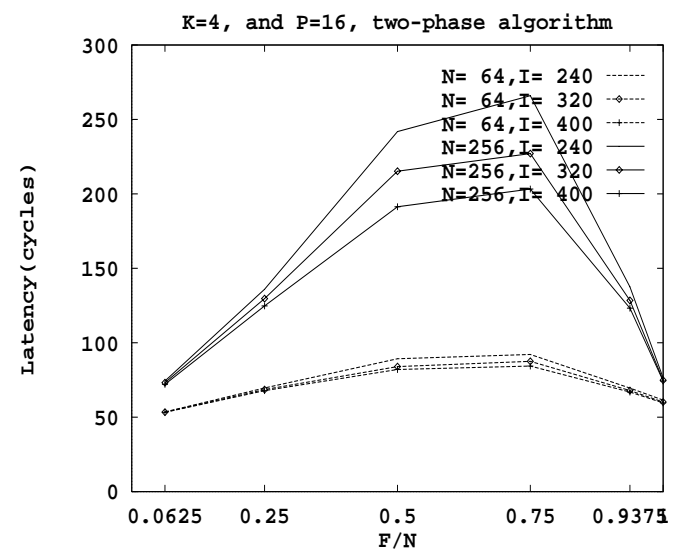

(f) Impacts of network size : latency vs. (fanout)/(network size) for two-phase multicast algorithm

Figure 4. The simulation results 
algorithm is much less than that of $\mathrm{u}$-min, and that is bounded approximately under the latency of multicast of fanout $2^{m}$ where $m$ is the minimum integer satisfying $2^{m} \geq \sqrt{N}$. Figure 4.(f) shows that when $N=256(64)$, the broadcast latency is similar to the multicast latency of fanout 16(8) in the network, as expected by Partition algorithm.

\section{Conclusions}

This paper proposed two-phase multicast algorithm in pure wormhole-switched bidirectional banyan networks where a $2 k \times 2 k$ switching element has synchronous replication logic and a bidirectional port has an one-flit output buffer. We compared the proposed algorithm with other by simulation in terms of multicast latency and multicast packet overhead.

To get high performance without deadlock, two-phase multicast algorithm solved multicast issues with the viewpoint of multicast latency. The algorithm requires only two transmissions to send a multicast packet to an arbitrary number of desired destinations. The headers of phase I and II are encoded as $k$-ary cubes and desired destination addresses are coalesced into multiple $k$-ary cubes. Contention on output links is arbitrated by the most upper input link first scheme with periodic priority rotation.

As the number of desired destinations increases, the multicast latency and the multicast packet overhead of two-phase algorithm increase. However, they are bounded due to coalescing effects and also decreases when the number of desired destinations reaches above some value. The broadcast latency of two-phase algorithm is much less than that of $\mathrm{u}$-min, and is similar to the latency of multicast of fanout $2^{m}$ where $m$ is the minimum integer satisfying $2^{m} \geq \sqrt{N}$. This is noticeable improvement on broadcast performance.

As a result, two-phase algorithm significantly outperforms than other scheme. The architectural support for twophase algorithm is so simple to be suitable for constructing large-scale multicomputer systems. Thus, two-phase algorithm can supports efficient multicast and broadcast in large-scale multicomputer systems.

\section{Acknowledgement}

J. Park's work is supported in part by KOSEF(Korea Science and Engineering Foundation) through Center for Artificial Intelligence Research at Korea Advanced Institute of Science and Technology.

\section{References}

[1] C.B.Stunkel and et al. Architecture and implementation of Vulcan. In Proc. of the Int'l Parallel Processing Symposium, pages 268-274, Jun. 1995.
[2] R. C.B.Stunkel and D.K.Panda. Implementing Multidestination Worms in Switch-Based Parallel Systems: Architectural Alternatives and their Impact. In Proc. of the 24th ACM Annual Symposium on Computer Architecture, pages 50-61, June. 1997.

[3] C.-M. Chiang. Multicasting in Multistage Interconnection Networks. PhD thesis, Dept. of Comp. Sci., Michigan State Univ., East Lansing, MI, 1995.

[4] I.D.Scherson and D.-H.Chien. Least Common Ancestor Networks. In Proc. of the 7th Int. Parallel Processing Symposium., pages 507-513, 1993.

[5] C. E. Leiserson and et al. The Network Architecture of the Connection Machine CM-5 . In Proc. of the ACM Symposium on Parallel Algorithms and Architectures, pages 272-285, 1992.

[6] L.M.Ni and P.K.McKinley. A survey of wormhole routing techniques in direct networks. IEEE Computer, 26:62-76, Feb. 1993.

[7] M. Meiko Limited, Waltham. Computing Surface: CS-2. In Communications Networks, Jun. 1993.

[8] N.Nupairoj and L.M.Ni. Issues in Designing Efficient and Practical Algorithms for Collective Communication in Wormhole-Routed Systems. In 1st International Workshop on Communication and Architectural Support for NetworkBased Parallel Computing, pages 212-226, Feb 1997.

[9] D. K. Panda and R. Sivaram. Fast Broadcast and Multicast in Wormhole Multistage Networks with Multidestination Worms. Technical Report OSU-CISRC-4/95-TR21, Dept. of Comp. and Info. Sci., Ohio State Univ., 1995.

[10] H. Xu, Y.-D. Gui, and L. M. Ni. Optimal Software Multicast in Wormhole-Routed Multistage Networks. In Proc. of Supercomputing, pages 1252-1265, Dec. 1994. 